\title{
Iuri Lotman: \\ Interacciones entre semiótica y educación
}

\section{Iuri Lotman: \\ Interactions between semiotics and education}

\author{
DOI: http://dx.doi.org/10.17981/cultedusoc.11.1.2020.10 \\ Recibido: 09/11/2019. Aceptado: 19/02/2020. Publicado: 23/03/2020 \\ Elennys Oliveros-Rodríguez \\ Universidad Surcolombiana. Neiva (Colombia) \\ elennysoliveros@yahoo.com
}

Para citar este artículo:

Oliveros-Rodríguez, E. (2020). Iuri Lotman: interacciones entre semiótica y educación. Cultura, Educación y Sociedad, 11(1). 139150. DOI: http://dx.doi.org/10.17981/cultedusoc.11.1.2020.10

\section{Resumen}

El presente artículo de reflexión tiene como propósito exponer los aportes y postulados de la semiótica de Iuri Lotman. Para esto, primero se hace un recorrido por la noción de semiótica y sus interrelaciones con otros campos del conocimiento, así como los elementos de confluencia entre esta disciplina y los préstamos terminológicos que ha recibido de áreas como la química y la biología, para finalmente entrar en la discusión de la semiótica como una metaciencia. Luego, se discuten algunos de los postulados de la semiótica lotmaniana, los cuales se sostienen en nociones como semiosfera, texto, frontera y semiosis. Se concluye la reflexión con los aportes de la semiótica en la educación y el importante papel de las operaciones semióticas de atribución, sustitución y superación para explicar cómo durante el proceso de enseñanza y aprendizaje el discente intérprete productor identificará nuevos valores gracias al proceso de atribución y sustitución de lo que ya sabía.

Palabras clave: semiótica; semiosfera; educación

\begin{abstract}
The present reflection article has as purpose to expose the contributions and postulates of Iuri Lotman's semiotics. To this end, it first reviews the notion of semiotics and its interrelations with other fields of knowledge, as well as the elements of confluence between this discipline and the terminological borrowings it has received from areas such as chemistry and biology, to finally enter into the discussion of semiotics as a meta-science. Then, some of the postulates of Lotmanian semiotics are discussed, which are sustained in notions such as semiosphere, text, border and semiosis. The reflection concludes with the contributions of semiotics in education and the important role of the semiotic operations of attribution, substitution and overcoming to explain how during the process of teaching and learning the student interpreter-producer will identify new values thanks to the process of attribution and substitution of what he already knew.
\end{abstract}

Keywords: semiotics; semiosphere; education 


\section{INTRODUCCIÓN}

En la obra de Ferdinand de Saussure, la semiología representa una ciencia más amplia que la lingüística, cuyo propósito es estudiar el conjunto de signos que los hombres utilizan (Saussure, 1972). Otros autores como Barthes citado por Mounin (1972) señalan que la semiótica (semiología) es la parte de la lingüística que estudia "las grandes unidades significantes del habla" (p. 13). En su libro Fundamento de la teoría de los signos, en la nota terminológica que precede al trabajo de Morris, se puede leer que el vocablo semiótica significa "doctrina de los signos" (Mounin, 1972). Por otra parte, Peirce también citado por Mounin (1972), usó este término según la acepción que le otorgó Locke para definir a una cuasiformal doctrina de los signos, mientras que Saussure (1972) hace referencia a "una ciencia que estudia la vida de los signos" (p. 17).

Como se ha expuesto, se plantean diversas posturas epistémicas en relación con lo que es el objeto de estudio de la semiótica. En otras palabras, los campos de interés de la semiótica son variados, como variadas también son las conceptualizaciones y las teorías en torno al signo, debido a que cada teórico construye su sistema conceptual que puede estar sustentado sobre la base de modelos formales, sobre la base de la cultura y la comunicación o hacia la construcción de modelos biológicos de organización (Vidales, 2011).

Para Eco (1994), la semiótica es la disciplina que estudia las posibles variedades de los signos. Aunado a esta definición, agrega que esta disciplina puede emplearse como técnica de investigación dado que busca explicar de manera bastante precisa "cómo funcionan la comunicación y la significación” (p. 12). Aparte considera que, la semiótica se encarga del estudio de las relaciones entre el código y el mensaje, "y entre el signo y el discurso." Añade, además, que la materia prima de esta ciencia son los signos, pero no los signos en completo aislamiento, sino en relación con los códigos y con unidades más amplias como por ejemplo "el enunciado, la figura retórica, la función narrativa" (Eco, 1994, p. 19).

Eco insiste en que la semiótica no es solo una teoría, sino también constituye una forma de la praxis. Para otros autores, la semiótica se refiere a una teoría de los signos y al análisis de los sistemas de signos o señales utilizados para la comunicación (Richards, Platt y Platt, 1997). En otras palabras, la semiótica caracteriza a las lenguas naturales no en sí mismas, sino como objetos para estudiar y comparar con otros sistemas de signos, lo que le permite definir algunos rasgos de las lenguas y establecer tipologías (Simone, 2001).

De acuerdo con Morris (1985), la semiótica "tiene un doble vínculo con las ciencias: es una ciencia más, y a la vez un instrumento de las ciencias" (p. 24). Blanco (2006) por su parte, sostiene que la semiótica nace en la confluencia de la lingüística, la antropología y en su momento recibió la influencia del formalismo estructuralista, lo que da cabida a nociones como cuadro semiótico, recorrido generativo, esquemas y programas narrativos.

Con el paso de los años, la semiótica se ha visto influida por el aporte que realizan al conocimiento otras ciencias humanas, sin dejar de lado la contribución que ha recibido de las ciencias naturales. Continúa Blanco que, de la química, la semiótica tomó los términos de isotopía y de valencia; este último, recibido a través de la lingüística estructural. Este tipo de préstamos a otras ciencias lo realiza también Lotman, puesto que este autor "acoge y explota nociones procedentes de las ciencias matemáticas y naturales ("isomorfismo", "asimetría”, "entropía”, “irreversibilidad”, la oposición "discreto/continuo”, etc.) y emplea analogías 
explicativas inspiradas en los dominios de la biología, de la química y de la física» (Lampis, 2015, p. 400).

Para Morris (1985), la semiótica presenta una doble vinculación con las ciencias: es una ciencia en sí y puede ser empleada como instrumento de análisis para las otras ciencias, puesto que "cada ciencia utiliza y expresa sus resultados por medio de signos" (p. 25), de allí que se hable de la semiótica como una metaciencia. Lo que establece esta conjunción entre disciplinas de distinto orden es, precisamente, la noción que se maneja de signo. Es este elemento el que logra la cooperación entre las ciencias sociales, las psicológicas y humanísticas, pero también en las ciencias biológicas y físicas, las cuales, no solo se sirven de los signos lingüísticos propiamente, sino de los signos entendidos como procesos, fenómenos, construcciones.

Otra de las disciplinas con las que tiene relación la semiótica es la comunicación. Esta colaboración o diálogo se sustenta en los aportes de la semiótica a la comprensión del proceso de comunicación, entendido más allá de una mera transmisión de mensajes, sino como un proceso de producción de sentido y de intercambio simbólico signados por la cultura. La semiótica también ha sido empleada como herramienta de análisis para el estudio de la comunicación de masas, así como para la comunicación no verbal (Vidales, 2009; Castillo, 2017).

Como se ha expresado supra, la semiótica es una metaciencia que desde sus diversas genealogías, sistemas conceptuales y métodos heurísticos permite no solo estudiar el signo lingüístico como objeto central en los procesos de comunicación, sino además ha desarrollado modelos de análisis que ven en el texto, el anuncio, el film, el hipertexto, la celebración eucarística, la clase, entre otras prácticas significantes, un "espacio antroposemiótico complejo de múltiples relaciones" (Vidales, 2011, p. 156), el cual está incluso por encima de la noción de signo, que había sido entendida como unidad nuclear para el análisis.

La semiótica dialoga también con la pedagogía, puesto que la educación es un fenómeno social, de construcción y asignación del sentido por medio de prácticas significantes que llevan a término tanto docente como discente, ambos, "sujetos sensibles, cognoscentes e históricos reflexivos en constante formación" (García, 2014, p. 84). Estas prácticas significantes implican desde saludar, enseñar, presentar disculpas, proclamar un discurso o exponer un trabajo de investigación en algún congreso, con lo cual estamos comunicando nuestra subjetividad a otros y todos estos procesos de significación se llevan a cabo gracias al uso de variados códigos semióticos.

\section{Desarrollo}

\section{La semiótica colabora con la arquitectura}

Investigaciones como las que llevaron a cabo Lara-Escobedo, Rubio-Toledo e Higuera-Zimbrón (2011) se basaron en una metodología semiótica para interpretar la experiencia del usuario con respecto a los espacios arquitectónicos. Así entendido, los usuarios son quienes les asignan un significado a las formas espaciales de los edificios o a los espacios arquitectónicos de una ciudad, puesto que todo se ha diseñado para sugerir, para provocar en los espectadores determinados comportamientos. Los autores indican que "la discusión se aborda desde la semiótica, como una especie de modelo teórico, del cual no puede, ni debe prescindir ninguna disciplina" (p. 140). 


\section{La semiótica de Iuri Lotman}

Iuri Lotman nació en 1922 (Leningrado, Estonia) y murió en 1993 en Tartu (Estonia). Fue profesor de la Universidad de Tartu-Estonia desde la década del 50 hasta su muerte. Egresado de Literatura y Filología de la Universidad Estatal de Leningrado (San Petersburgo, Rusia). Entre sus obras se encuentra: Lecciones de poética estructural, Trabajo de los sistemas de signos, Estructura del texto artístico, Estética y semiótica del cine, Semiótica de la cultura, La semiosfera, Acerca de la Semiosfera, Cultura y Explosión y Semiosfera III, y Semiótica de las artes y de la cultura (Cáceres citado por Lotman, 1996).

Lotman fue miembro de la Comisión Internacional de Semiótica y de la Asociación Americana de Semiótica, miembro del Consejo de Ciencias Sociales de la Unesco; distinciones que confirman su imagen como la figura más notable de la semiótica de mediados del Siglo XX. Recibió el Doctorado Honoris Causa de la Universidad de Bruselas (Bélgica) y de la Universidad Carolina de Praga (República Checa). Su trabajo investigativo ha recibido una amplia acogida entre la comunidad académica, no solo de Rusia, sino de otros países, lo cual ha llevado a la traducción de su obra a más de 20 idiomas. Se le considera el semiótico más influyente en la segunda mitad del siglo XX.

Según Cáceres (citado por Lotman, 1996), entre 1964 y 1974 se gesta la Escuela Semiótica de Tartu-Moscú. En este período, esta escuela se interesa en estudiar temas relativos a la lingüística estructural. También se interesaron en hallar analogías entre el sistema lingüístico, las lenguas naturales y los sistemas de signos del mito y del arte. Entendieron los sistemas modalizantes primarios como una estructura de comunicación que se encuentra en un nivel superpuesto al de los sistemas lingüísticos, lengua natural o sistema primario "y que, en cuanto a sistemas semióticos, son considerados como modelos que explican al mundo" (Cáceres citado por Lotman, 1996, p. 25).

Sebeok (2005) reflexiona sobre el postulado los modelos del mundo, a través de las nociones sistema modalizante primario y sistema modalizante secundario. Entiéndase un sistema modalizante como un lenguaje, el cual puede ser natural, es decir, un sistema de signos que se adquiere en la interacción social y dentro de una cultura, o, un lenguaje artificial construido sobre el modelo de la lengua natural. Pueden existir sistemas que poseen un lenguaje natural de base, los cuales soportan superestructuras de segundo orden que a su vez crean lenguajes de segundo nivel a los que denominó Lotman como sistemas modalizantes secundarios. Estos corresponden a los lenguajes del arte, el mito, la religión, la música, ya que se construyen y modelan desde una lengua natural (Cáceres, 1994).

El lenguaje natural es básico, primario y aporta la infraestructura para que tengan asidero los otros sistemas humanos de signos. En estos primeros años, Lotman, o mejor, la Escuela de Tartu, se interesó por los estudios literarios, cuyas investigaciones se publicaron en los primeros números de Trabajos sobre los sistemas de signos. Navarro (citado por Lotman, 1996) reflexiona como entre los aspectos novedosos de este teórico está la concepción del texto y de la cultura como generadores de sentido, más que como depósitos.

Por otra parte, Lotman (1996) reconoce que "sin semiosfera el lenguaje no sólo no funciona, sino que tampoco existe" (p. 20). Como es posible inferir, el constructo semiosfera permite ver cada proceso semiótico como un continuum que va desde las señales emitidas por los satélites, los versos, el ruido de los animales y las lenguas naturales como un gran espacio semiótico en el que todo está interconectado. 


\section{La semiosfera de Iuri Lotman}

En la obra de Iuri Lotman, el concepto de semiosfera es fundamental. Para este autor, la semiosfera se concibe como un continuum, de carácter abstracto ya que en sí misma es un gran sistema, "un espacio semiótico fuera del cual es imposible la existencia misma de la semiosis" (Lotman, 1998, p. 12). Con esto el autor quiere establecer que solo en la semiosfera se lleva a cabo la semiosis, el sentido. Este es el espacio donde se mueve la actividad docente.

Aunque posea un carácter cerrado, esta característica o rasgo distintivo como lo denominó Lotman, define a la semiosfera como un lugar que evita todo contacto con los denominados textos alosemióticos o con los no-textos. Para eludir el contacto con los textos alosemióticos, la semiosfera requiere de una frontera, noción que ha creado Lotman para indicar la traducción de un lenguaje o los lenguajes que a través de los filtros y los traductores bilingües con los que cuenta la frontera pueden pasar de una semiosfera a otra.

Otro de los aspectos importantes de la noción semiosfera, es la división que se establece entre núcleo y periferia que vienen a formar parte de una organización interior a la semiosfera, y por tanto se constituyen en una ley. De acuerdo con esta ley, el núcleo está constituido por los sistemas semióticos dominantes. Por su parte, las formaciones semióticas de la periferia están representadas no solo por estructuras cerradas (lenguajes), "sino por fragmentos de los mismos, o incluso por textos aislados” (Lotman, 1998, p. 17).

La noción de periferia permite establecer que, con un pedazo de una estructura semiótica, ya desaparecida, se puede recuperar o reconstruir todo un sistema, dado que el texto aislado en sí mismo contiene los mecanismos para generar la reconstrucción del sistema semiótico.

La semiosfera también presenta fronteras internas, cuya función es la de especializar determinados sectores desde un punto de vista semiótico. Como lo expresa Lotman (1998): "La transmisión de información a través de estas fronteras, el juego entre diferentes estructuras y subestructuras (...) determinan generaciones de sentido, el surgimiento de nueva información" (p. 17). Por otro lado, se hace necesario explicar que existe una diversidad interna de la semiosfera, y, por tanto, cada parte de ella es tratada como un órgano dentro de un organismo.

Con base en lo expresado, queda claro que la frontera del espacio semiótico es una noción muy importante dada su "posición funcional y estructural que determina la esencia del mecanismo de la misma" (Lotman, 1998, p. 13). Solo con la ayuda de la frontera, la semiosfera puede vincularse con los espacios alosemióticos, porque la frontera es un mecanismo bilingüe que "traduce los mensajes externos al lenguaje interno de la semiosfera y a la inversa" (Lotman, 1998, pp. 13-14).

En ese sentido, Lotman caracteriza la semiosfera como un continuum, un gran sistema, un espacio semiótico donde tiene lugar la semiosis, aunque posee un carácter delimitado que se refleja en la noción de frontera. De igual modo, la semiosfera da cuenta de una irregularidad semiótica, debido a que presenta estructuras nucleares y estructuras de periferia y, por ende, se violan "las jerarquías de los lenguajes y de los textos" (Lotman, 1998, p. 16). También la semiosfera da cuenta de una profundidad diacrónica, debido a que presenta un gran sistema de memoria, sin el cual no puede operar. En otras palabras: sin la semiosfera el lenguaje no existe. 


\section{Semiótica y educación}

En los procesos de construcción y apropiación de los signos se observan dos momentos: la semiosis sustituyente y la semiosis sustitutiva. Entendemos por semiosis sustituyente al conjunto de "configuraciones perceptuales (frases, imágenes, objetos y comportamientos exhibidos)" (Magariños, 2008, p. 63) con los que se le asigna un sentido a lo que se percibe en el entorno o en las personas que las utilizan. Por ejemplo, el docente para hacer partícipe al discente de la semiosis sustituyente, se basa en las secuencias didácticas, las cuales son de carácter intencional; además, utiliza los recursos educativos internos y externos que sostienen el acto de enseñanza y lo enriquecen para configurar en la mente de este intérprete productor (discente), el conjunto de esquemas necesarios para la producción de la semiosis.

De este modo, los aprendizajes significativos tienen lugar cuando el docente productor intérprete logra desarticular los constructos semióticos de los discentes intérpretes productores (García, 2014), a través de lo que se denomina semiosis sustituida, la cual se logra si el docente productor intérprete permea por medio del discurso pedagógico y de las estrategias concebidas para tal fin, las concepciones que presentan los estudiantes sobre determinados objetos del mundo.

Durante estos procesos de enseñanza y aprendizaje se transita por las fases de atribución, sustitución y superación (Magariños, 2008), tres operaciones semióticas sumamente imprescindibles y con las que el discente intérprete productor identificará nuevos valores gracias al proceso de atribución y sustitución de lo que ya sabía. El propósito de estas etapas no es otro que alcanzar la resignificación del mundo, la adquisición del conocimiento validado y operativo que le permite al estudiante comprender las situaciones, además de estar preparado para crear o deconstruir otros mundos semióticos posibles. En otras palabras, las operaciones de atribución, sustitución y superación pueden ser vistas en sí mismas como marcadores importantes para reconocer si hubo o no aprendizaje significativo. Al respecto, investigadores como Luque y Marín (2001) y también Mena y Huneeus (2017), destacan la importancia de integrar la teoría y la práctica como vía para fortalecer los aprendizajes.

Según García (2012), la metodología que propone la semiótica cognitiva ayuda a esclarecer los fenómenos de aprehensión de nuevos esquemas de pensamiento en los estudiantes; instaura una nueva mirada respecto a cómo debe ser tratado el sujeto en el acto educativo y con ello da cuenta, no solo, de un docente productor intérprete, dueño del conocimiento, sino que también ofrece luces sobre de qué depende la interacción que se da con el discente intérprete productor. De esta manera, en esta semiótica cognitiva dialéctica se entiende al estudiante como un sujeto sensible al flujo de la información que produce la semiosfera y que lo afecta en sus creencias y conocimiento del mundo.

García (2012) explica que los aprendizajes significativos solo se logran si participan y se hacen explícitos en la práctica docente, las denominadas operaciones semióticas "fundamentales que tratan determinadas opciones de pensamiento, proyectadas a mundos semióticos posibles, en el marco de una teoría constructivista de los aprendizajes" (García, 2012, p. 13). En esta medida, el discente, desde los espacios semióticos de interacción, colabora en la apropiación de nuevos modelos de conocimiento. 
Desde esta perspectiva, el reconocimiento de la noción de mundo, como espacio de lo cognoscible, resulta relevante también para entender de qué modo se pueden adecuar las variadas prácticas significantes a la actividad situada (García, 2012), es decir, a la aplicación de estrategias didácticas que apoyen el proceso de traducción de nuevas formas semióticas en el campo formativo.

En este contexto, la metodología propuesta por la semiótica cognitiva dialéctica tiene el propósito de colaborar en el mejoramiento razonado del hecho educativo, sobre todo en lo que atañe al reconocimiento de las distintas semiosis que coocurren en el aula de aprendizaje, así como aquellas semiosis sujetas a las concepciones, supuestos y modos de ver el mundo del sujeto discente. El docente productor intérprete, en su rol de traductor entre los sistemas modalizantes primarios y secundarios, necesita conocer cómo operan estas semiosis para desentrañarlas, comprenderlas e integrarlas al hecho educativo a través de la praxis dialéctica y de los recursos didácticos disponibles, en función siempre de esa armonía necesaria entre el currículo y la identidad cultural de los estudiantes.

El papel del maestro como traductor de la semiofera no se limita solo a la frontera lotmaniana; su impacto y ejercicio eficaz debe sostenerse sobre la noción de pensamiento, categoría teórica íntimamente ligada al constructo lotmaniano de conciencia. La significación parece estar prendida a esta noción, ya que, sin conciencia, la semiosis no ocurre, no tiene lugar. Así, no solo existe el pensamiento científico, aquel que se requiere para la producción de conocimiento acreditado por una comunidad de investigadores, sino que, también se requiere, en sintonía con este, de un pensamiento lúdico, creador, artístico, con el propósito de promover nuevas concepciones y nuevos procesos de significación en el aula.

En este contexto de producción de semiosis, el pensamiento sufre transformaciones en sus límites, debido a que es un "sistema virtual de interpretación, por la eficacia de la inclusión de un nuevo interpretante, que, construido a partir del enunciado emergente de determinada semiosis" (Magariños, 2007, p. 98) establece un nuevo objeto para percibir a través de la razón, y por qué no, de los sentidos. En este proceso de las primeras percepciones, se hace necesario explicar la noción de semiosis, entendida como un sistema virtual de signos de diverso orden (indicio, símbolo, ícono) y sus relaciones y combinaciones, a partir de las cuales surgen las expresiones semióticas con las que los sujetos, la comunidad que hace uso de estos signos, establece una configuración "visual, comportamental, conceptual” (García, 2012, p. 7) de lo que la circunda.

Los signos creados a través de la semiosis son arbitrarios, aunque algunos semióticos han explicado que pueden ser motivados. Estos signos pueden ser estudiados desde distintas disciplinas lingüísticas, tales como la sintaxis, la fonología o la semiótica o las semióticas encargadas de analizarlos en sus contextos de producción. Asimismo, la noción de mundo es crucial para los investigadores, pues en ella se posicionan los sujetos productores e intérpretes de signos, con sus dificultades en la organización de sus prioridades y anhelos, lo cual provoca nuevas semiosis, que, dado su origen, podrán ser semiosis sustituyente o semiosis sustituida. La primera implica un conjunto de configuraciones propias del mundo cultural y por extensión, del individuo. Esta semiosis está sujeta a ser sustituida por otra en cualquier proceso de producción de aprendizajes significativos, lo que aplica como dos modelos o momentos en la configuración del significado, y más adelante, del sentido, de allí que entre la didáctica en esta relación. 
La semiótica y la didáctica entonces se vinculan por las herramientas heurísticas, por un metalenguaje que permite adentrarse en el heterogéneo conjunto de interacciones que el sujeto, centro del hacer educativo, recibe de su entorno y de los múltiples textos de los que se sirve para aprender. La didáctica por su parte, pone al servicio una serie de estrategias y actividades para ayudar a este sujeto discente que necesita apropiarse de los conocimientos, a partir de la mediación de un docente-traductor, en el complejo y dinámico mundo de la educación.

El docente, como traductor en la semiosfera, es un colaborador en la construcción de sentido por medio de las semiosis sustituyentes. A través de un proceso dialógico, media entre lo que conoce el discente y entre las fronteras que separan los textos semióticos de los alosemióticos, dentro de las dinámicas propias de la cultura. Enseña lo que es aceptado como núcleo de la semiosfera y lo que forma parte de su periferia. El aula, como espacio de lo cognoscible, el currículo como modelo ideal de lo aprehensible, requiere que el docente filtre, modele, interprete y traduzca las nuevas formas semióticas, sirviéndose de un lenguaje que le permita alcanzar los objetivos formativos y la semiotización de los elementos alosemióticos que habían estado excluidos del hecho didáctico. Entendido de este modo, el aula de clase es solo uno de los diversos contextos en los que el discente intérprete productor de signos participa de la semiosis.

García (2012) establece esta relación de necesidad entre la semiótica y la didáctica cuando señala que como ser social integral, el hombre se halla en la constante búsqueda de formación, porque en sí misma, la educación forma parte del eje de construcción de ese hombre moderno dispuesto a cultivar numerosas habilidades que le hagan aún más hábil para pasar de una semiosfera a otra: del campo o semiosfera de la comunicación comercial a la semiosfera de las relaciones de venta, promoción e información de productos, por ejemplo.

La semiótica le ofrece a la didáctica el campo de interpretación para entender que en el proceso educativo ocurren continuamente diversas semiosis, y es a través del acto de compartir significados con otros y traducirlos, en el sentido lotmaniano, cuando se reconoce que la semiótica “...es una práctica significante, científica y socializante en cuyo ejercicio se puede comprender su naturaleza y alcance como disciplina de las ciencias humanas.“ (García, 2012, p. 13).

La semiótica comparte finalmente con la didáctica el hecho, necesario, por cierto, de participar en el estudio, en la observación de un continuum que se va modificando en la medida en que se van introduciendo nuevos cambios en el modo de concebir las variaciones en la significación, no solo en el contexto de la cultura, sino en el aparato crítico que se emplea para estudiar estas variaciones que van desde un espacio textual, social, hasta llegar a la semiosfera. Este modo de comunicación entre ambas disciplinas se ve triangulado en el sujeto discente, que en este caso asume esas variaciones, finamente observables incluso en los medios de apropiación del conocimiento, para ser capaz de traducirlas, filtrarlas y construir a través de ellas su visión del mundo, su visión del constructo que está aprendiendo a reconocer, del que se está apropiando.

Lo ideal sería que esta relación entre semiótica y educación fuese más explícita en el aula, ya que el discente necesita reconocer las etapas de aprehensión del conocimiento, que extrapolándolo a la teoría de Magariños de Morentin se entiende como atribución, sustitución y superación (Magariños citado por García, 2012). 
La semiótica es central en el estudio de la producción del sentido, lo que la hace una ciencia que puede moverse más allá de su campo y colaborar con otras disciplinas. Tanto la didáctica como la semiótica son disciplinas, metodologías que ayudan a comprender determinados contextos. Se requieren mutuamente, dado que por un lado la didáctica posee los instrumentos teóricos y metodológicos para dar cuenta de la naturaleza del fenómeno educativo, así como de sus causas. Por el otro, la semiótica como metodología ayuda a explicar el complejo mundo de producción e interpretación de los signos.

Como bien apunta García (2012), la educación se preocupa por la formación integral del ser humano, en la transformación de este sujeto que proviene de ambientes en los que prevalece un sistema modalizante primario, con sus costumbres, concepciones particulares del mundo, con sus propias semiosis, para luego, a través de las didácticas y de otras ciencias auxiliares, cultivar el carácter del discente en función de un sistema de conocimiento, según las necesidades de un mundo cada vez más competitivo.

Los movimientos o cambios de actitud ante determinados constructos, objetos semióticos o fenómenos pueden ser descritos con la mayor precisión que les otorga a las ciencias humanas el campo de los estudios semióticos. Tal y como lo expone García (2012), estos cambios se dan desde operaciones fundamentales como la atribución, la sustitución y la superación planteadas desde la semiótica dialéctica cognitiva, y que implican "verdaderos cambios mentales en los sujetos que experimentan ese conjunto de acciones" (García, 2012, p. 5).

El conocimiento de estas operaciones semióticas no solo permite observar y seguir los cambios cognitivos en los discentes, sino sopesar de primera mano la aceptación de la nueva información y su transformación en conocimiento práctico o teórico, según sea el caso. Como se sabe, la operación de atribución le aplica un valor relacional a cada forma semiótica, caracterizando con ello determinados objetos semióticos en función de los estímulos y relaciones perceptuales que estimulan el proceso en la mente del productor intérprete de signos. Durante la operación de sustitución, las formas semióticas reciben los valores de determinados universos, desde un plano sociohistórico en el que se hacen parte de las semiosis sustituyentes. Lo que se ve con esta operación es la comunicación entre los valores de las formas semióticas de un universo o semiosfera A, y los valores relacionales que estas formas adquieren en otro universo o semiosfera B (semiosis sustituida). En cuanto a la operación semiótica de superación, se le asignan nuevos valores a los pares semióticos que resultaron de las dos primeras operaciones semióticas (sustituyente y sustituida) (García, 2012).

En este sentido, es importante examinar nuevamente la definición de semiosfera en la obra de Iuri Lotman (1996; 1998). Para este estudios "la semiosfera es el espacio semiótico fuera del cual es imposible la existencia misma de la semiosis", es decir, en la semiosfera, nunca fuera de ella, se genera el sentido. El aula de lengua puede llegar a ser una semiosfera si el docente-traductor lo hace un espacio propicio o aplica los recursos que proporciona tanto la didáctica como la semiótica para construir escenarios en los que el estudiante llegue a sentirse a gusto y se dedique a aprender. Este proceso de construcción del aula de lengua en un espacio idóneo para la semiosis, pasa, primeramente, como especie de filtro, por el lenguaje que emplea el docente para comunicar o hacer discernibles los objetos semióticos a los estudiantes, es decir, para comunicar lo que sabe. 
El docente-traductor, en el sentido lotmaniano, es el llamado a encontrar las estrategias idóneas para determinados objetivos de aprendizaje. Si el lenguaje que utiliza para develar y mostrar el objeto, la cualidad u otro conocimiento es oscuro, codificado en una variedad lingüística que nada tiene que ver con el contexto en el que se forma el estudiante, difícilmente podríamos pensar que pueda darse la semiosis, o que el aula de lengua la propicie.

\section{Conclusiones}

El docente es un colaborador para la construcción de sentidos. Por medio de un proceso dialógico media entre lo que el alumno conoce, con otros niveles de la semiosfera. Se ha dicho que el aula de lengua necesita de un docente que filtre, modele, interprete y traduzca la información que conoce para el discente, sirviéndose de un lenguaje que le permita alcanzar los objetivos didácticos. El aula de lengua puede constituirse en una semiosfera si el sistema, el currículo y el docente logran hacer significativa esa memoria colectiva, esa historia viva de la que el niño es parte, dentro de un sistema modalizante primario, y el maestro, en su rol de traductor de la semiosfera y de los sistemas modalizantes secundarios.

La educación en sí misma es una práctica significante porque a través del aparato heurístico y hermenéutico con el que cuenta, es capaz de propiciar en los ambientes escolares una simulación de las actividades comunes o especializadas que tienen cabida en la semiosfera de la cultura. Los objetivos que persigue la educación son siempre de orden semiótico; esto es, establecer o mover al discente a la adquisición de un conjunto de comportamientos que implican la aceptación de diversos significados, que no siempre están en concordancia o relación con las concepciones culturales de entrada.

La semiótica. por su parte, puede ayudar a esclarecer o hacer más viable ese tránsito entre contenidos ya sujetos, evaluados, sostenidos en la memoria a largo plazo de ese discente; ayudar en la asimilación de competencias que en sí mismas requieren un cambio en las estructuras cognitivas de los estudiantes, un cambio de comportamiento en los procesos de percepción y aceleración de las semiosis. La educación, aunque tiene una tarea muy trascendental, ya que debe estar en sinergia con los objetivos que persigue un currículo y un contexto global, requiere de la semiótica para poder dar el paso en la comprensión de cada una de estas prácticas significantes.

Allanar el camino en la adquisición de nuevos comportamientos semióticos, de nuevas formas de aprehensión de los sistemas modalizantes secundarios, cuyas convenciones y estructuras están supeditadas a las exigencias de cada campo disciplinar, es tarea de la educación, pero también de la semiótica. El paso de un conocimiento ya procesado, alojado en la memoria, arraigado en lo más hondo de la identidad del sujeto, a un conocimiento que rompe con los moldes o los esquemas previamente establecidos, no es solo el objeto de estudio de la psicología.

Finalmente, cuando el discente alcanza la operación semiótica de superación es porque ha sido capaz de relacionar los valores de las atribuciones de cada objeto semiótico del cual se sirve para describir segmentos de la realidad; ha aprendido a interpretar esa realidad en función de un contexto o una memoria colectiva y ha encontrado la manera de conectar estas nuevas formas semióticas en el continuum dinámico de la cultura. 
En definitiva, se ha movido de un plano meramente descriptivo, histórico, a un plano interpretativo, relacional y transtextual, más en relación con lo que Lotman denominó la semiosfera de la cultura. Cuando el discente alcanza, mediado por una propuesta didáctica, la última operación semiótica, podemos considerar, sin ambages, que ha alcanzado el aprendizaje significativo, el cual le será útil para toda la vida.

\section{REFERENCIAS}

Blanco, D. (2006). Semiótica y Ciencias Humanas. Revista Letras, 77, 111-112. Recuperado de http://www.acuedi.org/ddata/3157.pdf

Castillo, M. (2017). ¿Semiótica en el estudio de la comunicación? Razón y palabra, 21(4_99), 34-44. Recuperado de https:/www.revistarazonypalabra.org/index.php/ryp/article/ view/1074

Cáceres, M. (1994). Lenguaje, cultura, semiosfera. En, J. Paz, J. Fernández y C. Gómez (cords.), Actas del V Congreso internacional de la Asociación Española de Semiótica, Semiótica y modernidad (pp. 129-136). Universidade da Coruña, Coruña. Recuperado de https://core.ac.uk/download/pdf/61903492.pdf

Eco, U. (1994). Signo. Barcelona: Labor.

García, Í. (2014). Pedagogía semiótica sensible en la práctica significante de la docencia. Ontosemiótica, 1(1), 77-86. Recuperado de http://erevistas.saber.ula.ve/index.php/ontosemiotica/article/view/5354

García, Í. (2012). Semiótica y didáctica. Relaciones pensamiento/semiosis/mundo en la construcción de aprendizajes significativos en el Aula Preescolar. Omnia, 18(2), 11-24. Disponible en https://produccioncientificaluz.org/index.php/omnia/article/view/7389

Lampis, M. (2015). La teoría semiótica de Lotman y la dimensión sistémica del texto y de la cultura. Revista Signa, 24, 393-404. https://doi.org/10.5944/signa.vol24.2015.14727

Lara-Escobelo, M., Rubio-Toledo, M. e Higuera-Zimbrón, A. (2011). Semiótica y arquitectura. Quivera, 13(1), 139-155. Disponible en https:/quivera.uaemex.mx/article/ view/10162

Lotman, I. (1996). La semiosfera I. Semiótica de la cultura y del texto. Madrid: Cátedra.

Lotman, I. (1998). La semiosfera II. Semiótica de la cultura, del texto, de la conducta y del espacio. Madrid: Cátedra.

Luque, M. y Marín, F. (2001). Inserción de los proyectos pedagógicos de aula en las prácticas profesionales de la docencia. Multiciencias, 1(2), 117-128.Disponible en http:// produccioncientificaluz.org/index.php/multiciencias/article/view/16556

Magariños, J. (2008). La semiótica de los bordes. Apuntes de metodología semiótica. [Online]. Recuperado de http://www.magarinos.com.ar/Impresion.html

Magariños, J. (2007). La semiótica de los bordes. Tópicos de seminario, 2(18), 97-112. Disponible en http://www.topicosdelseminario.buap.mx/index.php/topsem/article/view/157

Mena, M. y Huneeus, M. (2017). Convivencia Escolar para el aprendizaje y buen trato de todos: Hacia una mejor comprensión del concepto. Cultura, Educación y Sociedad, 8(2), 9-20. https://doi.org/10.17981/cultedusoc.8.2.2017.01

Morris, Ch. (1985). Fundamentos de la teoría de los signos. Barcelona: Paidós.

Mounin, G. (1972). Claves para la lingüistica. México, D.F.: Anagrama. 
Richards, J., Platt, J. y Platt, H. (1997). Diccionario de lingüística aplicada y enseñanza de lenguas. Barcelona: Ariel.

Saussure, F. (1972). Curso de lingüistica general. Buenos Aires: Losada.

Sebeok, T. (2005). ¿En qué sentido es el lenguaje un sistema modalizante primario? AdVersusS, 2(2). Disponible en http://www.adversus.org/indice/nro2/articulos/articulo2Thomas\%20Sebeok.htm

Simone, R. (2001). Fundamentos de lingüística. Barcelona: Ariel.

Vidales, C. (2011). Semiótica y teoría de la comunicación. Tomo II. Monterrey: CECYTE, N.L.-CAEIP.

Vidales, C. (2009). La relación entre la semiótica y los estudios de la comunicación: un diálogo por construir. Comunicación y Sociedad, (11), 37-71. Disponible en http://www. comunicacionysociedad.cucsh.udg.mx/index.php/comsoc/article/view/1793

\footnotetext{
* Este trabajo es producto del seminario doctoral Semiótica y Educación, dirigido por la Dra. Irída García de Molero, en el marco del plan de formación en el Doctorado en Lingüística de la Universidad de Los Andes (Venezuela).
}

Elennys Oliveros-Rodríguez es docente tiempo completo de la Universidad Surcolombiana (Colombia). Investigador del Grupo IUDEX. 